\title{
Flebotomofauna al sureste del estado Lara, Venezuela
}

\author{
Luis Eduardo Traviezo \\ Sección de Parasitología, Universidad Centroccidental "Lisandro Alvarado", Barquisimeto, Venezuela.
}

Introducción. El reporte de 522 casos de leishmaniasis cutánea americana entre los años 1988 al 2002 en el estado Lara (específicamente al sureste donde hubo un repunte), motivó el estudio de los vectores implicados en la transmisión.

Objetivos. Determinar la abundancia, diversidad y distribución de flebótomos durante un año en 11 poblaciones situadas entre los 600 y 1600 metros sobre el nivel del mar, relacionando su abundancia con elementos climáticos.

Materiales y métodos. Para las capturas se utilizaron trampas CDC (18:00 a 06:00 horas) colocadas en zonas domésticas, peridomésticas y selváticas de casas donde hubo casos de leishmaniasis cutánea y se utilizó trampa Shannon en los alrededores de éstas, entre las 19:30 y las $22: 50$ horas.

Resultados. Se identificaron 10.326 ejemplares (8.867 hembras y 1.459 machos) con una diversidad de 11 especies, siendo Lutzomyia youngi (Feliciangeli \& Murillo 1987), la más abundante con un 96,54\%, seguida de Lutzomyia ovallesi (Ortiz, 1952) con 2,9\%, caracterizándose $L$. youngi por tener predilección por los ambientes peridomésticos y se apreció mayor actividad desde las 19:30 a las 20:30 horas (Shannon). La población de Las Maticas (1360 msnm) presentó la mayor abundancia, con un pico anual que coincidió con el final del verano, las primeras lluvias del invierno (régimen unimodal), la mayor temperatura media mensual del año $\left(24^{\circ} \mathrm{C}\right)$ y la menor humedad mensual del año $(70 \%)$.

Conclusión. Esta información permite conocer la población, ambiente y época del año donde se incremente la probabilidad de transmisión de la enfermedad, por la abundancia de las especies vectoras.

Palabras claves: Psychodidae, leishmaniasis cutánea, temperatura ambiental, Iluvias, humedad, Venezuela.

\section{Phlebotomine sandflies in the southeast of Lara state, Venezuela}

Introduction. The report of 522 cases of American cutaneous leishmaniasis between the years 1988 to 2002 in Lara State (specifically in the southeast where there was an increase of cases) prompted the study of the vectors species involved in transmission.

Objective. To determine the abundance, diversity and distribution of sandflies during one year in 11 populations located between 600 and 1600 meters above sea level and the relationship of their abundance to climatic elements.

Materials and methods. CDC traps placed in domestic, peridomestic and woodland areas around houses where cases of cutaneous leishmaniasis had been reported were used for the captures (18:00 to 06:00 hours) and Shannon traps were used in the environs of these houses between 19:30 to 22:50 hours.

Results: 10.326 specimens were identified ( 8.867 females and 1.459 males) with a diversity of 11 species, where Lutzomyia youngi (Feliciangeli \& Murillo 1987), was the most abundant species $(96.54 \%)$, followed by Lutzomyia ovallesi (Ortiz, 1952) with $2.9 \%$. L. youngi showed predilection for peridomestic areas and presented greater activity from 19:30 to 20:30 hours (Shannon). The population of Las Maticas (1360 meters above sea level) presented the highest abundance with an annual peak which coincided with the end of the dry period and the first rains of the rainy period (unimodal regime), the highest monthly average temperature $\left(24^{\circ} \mathrm{C}\right.$ ) and the lowest monthly humidity of the year $(70 \%)$. 
Conclusion. This information allows us to know the population, climatic variables and time of the year when the probability of transmission of the disease increases due to the abundance of the vector species.

Key words: Psychodidae, leishmaniasis cutaneous, temperature, rain, humidity, Venezuela.

Los estados Táchira, Mérida y Trujillo (Andes venezolanos) junto con el estado Lara concentran la mayor cantidad de casos de leishmaniasis cutánea americana de Venezuela; Lara ha sido en determinados años, el que ha aportado la mayor casuística en el territorio nacional. Tal fue el caso en el 2000 cuando se informaron 685 enfermos, más que los acumulados anualmente reportados en los 10 años anteriores; el municipio Andrés Eloy Blanco, al sureste del estado, presentó un aumento en el número de enfermos (110 casos) con sólo el 2,6\% de la población del estado (Biomedicina. Casos de leishmaniasis cutánea por distritos sanitarios. Comunicación personal. Venezuela, 2002), convirtiéndose en un área de importancia epidemiológica.

En Lara se han adelantado diversos estudios sobre la fauna de flebótomos asociada a leishmaniasis, comenzando con la descripción de Ortiz de tres especies nuevas: Lutzomyia ovallesi (Ortiz, 1952), Lutzomyia nuñeztovari (Ortiz, 1954) y Brumptomyia beaupertuyi (Ortiz, 1954) (Arredondo C. Flebótomos del Estado Lara. Memorias, III Simposio Venezolano de Leishmaniasis. Barquisimeto, 1987). Existe un vacío de información hasta 1972 cuando se comienzan a definir las especies presentes $(1,2)$ llegando a una diversidad que alcanza las 24 especies $(3,4)$; sin embargo, específicamente en el municipio Andrés Eloy Blanco, hasta el presente no se habían realizado trabajos.

Por esta razón, se realizó un estudio longitudinal de un año en poblaciones ubicadas a diferentes altitudes, con los objetivos de determinar la abundancia, diversidad y distribución de flebótomos en ambientes domésticos,

\footnotetext{
Correspondencia:

Luis Eduardo Traviezo, UCLA, Decanato de Medicina, Avenida Libertador con Avenida Andrés Bello, Apartado 400, Barquisimeto, Estado Lara, Venezuela.

Telefax: (0058) (251) 2591894.

Itravies@ucla.edu.ve

Recibido: 31/05/05; aceptado: 28/11/05
}

peridomésticos y selváticos, actividad en las primeras horas de la noche e infección natural de especies que pudieran estar comprometidas con la transmisión de la leishmaniasis cutánea y relacionar estos datos con variables climáticas.

\section{Materiales y métodos}

Área de estudio: el municipio Andrés Eloy Blanco se encuentra al sureste del estado Lara,

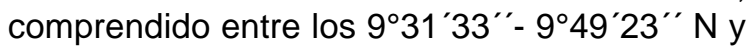
$69^{\circ} 20^{\prime} 26^{\prime \prime}-69^{\circ} 43^{\prime} 43^{\prime \prime}$ O, con una precipitación media anual de $826,81 \mathrm{~mm}$, una temperatura media anual de $22^{\circ} \mathrm{C}$ y una clasificación climática de subhúmedo seco frío; presenta relieves entre los 600 y los 2.000 msnm; los cultivos predominantes son el café y las papas.

Dentro del municipio se seleccionaron 11 puntos de captura sobre la base de la casuística de leishmaniasis cutánea americana, así: Las Virtudes (1.000 msnm), Sanare (1.200 msnm), Las Maticas (1.320 msnm), Monte Carmelo II (1.600 msnm) y Yacambu I (600 msnm), Yacambu II (800 msnm), Yacambu III (1.000 msnm), La Represa (1.000 $\mathrm{msnm})$, Periquito (1.200 msnm), La Escalera (1.250 msnm) y Monte Carmelo I (1.400 msnm). En estas últimas ocho poblaciones se hicieron capturas esporádicas (22 en total) ya que su ubicación presentaba problemas logísticos y de seguridad, mientras que en las cuatro primeras se hicieron capturas mensualmente capturas durante 12 meses (48 capturas, una captura/mes).

Métodos de captura: se colocaron tres trampas de luz CDC en cada población, la primera dentro de la casa seleccionada con casos de leishmaniasis cutánea (zona doméstica), la segunda en los alrededores de la casa, específicamente, donde estaban los gallineros (zona peridoméstica) y la tercera en zonas boscosas a unos $30 \mathrm{~m}$ de las casas seleccionadas (zona selvática). Estas trampas CDC se encendían a las 18:00 horas y se recogían a las 07:00 horas del siguiente día.

También se utilizaron trampas de luz Shannon pero sólo en Las Virtudes, Sanare, Las Maticas y 
Monte Carmelo II; la misma se colocaba a $30 \mathrm{~m}$ de viviendas donde se habían presentado casos de leishmaniasis cutánea americana. Estas trampas se encendían de las 19:30 a las 20:30, se apagaba y se agitaba, volviéndose a encender desde las 20:40 hasta las 21:40 horas; se apagaba y se encendía nuevamente de las 21:50 a las 22:50; estos intervalos de 10 minutos eran para garantizar que los flebótomos atrapados no correspondían a los atraídos de la hora anterior.

Transporte, montaje e identificación: los flebótomos recolectados se colocaban en jaulas con cubiertas de organdí, dentro de cavas de poliuretano con alta humedad para el traslado al laboratorio donde eran adormecidos con éter y, luego, colocados por separado en cápsulas de Petri con solución de detergente al $0,5 \%$ para remover los pelos y disminuir la tensión superficial; posteriormente, los machos se colocaron en solución clarificadora de Nesbitt, durante 24 horas para montarlos, luego, en solución de Berlese y precisar las características morfológicas de la genitalia y ascoides para compararlas con los descritos en las claves $(3,4)$; en las hembras se examinaron las espermatecas y la armadura del cibario según claves citadas.

Determinación de infección natural: para determinar la infección natural con flagelados, se retiró la cabeza del cuerpo y se separó el tracto digestivo entero para, luego, examinarlos al microscopio (600X). Los posibles flagelados se identificaron según el lugar de ubicación en el intestino para, posteriormente, en caso de hallar ejemplares infectados, aspirar con jeringa de tuberculina e inocular en cultivos y en hámster.

Características climáticas: se analizaron los datos hidrometeorológicos de cuatro estaciones climáticas ubicadas en el municipio Andrés Eloy Blanco (5) con el fin de conseguir la relación entre la abundancia de flebótomos y la oscilación de la temperatura media mensual, la humedad y la precipitación acumulada mensual de la zona de captura.

\section{Resultados}

Se evidenció la presencia de ejemplares de dos géneros de Lutzomyia (França, 1924) y Brumptomyia (França y Parrot, 1921); el género Lutzomyia presentó mayor abundancia y diversidad de especies, 11, de las cuales, Lutzomyia youngi (Feliciangeli \& Murillo 1987), fue la más abundante con 9.749 ejemplares $(95,4 \%)$ de los 10.217 recolectados, seguido de $L$. ovallesi con $3,72 \%$ (380), de menor abundancia (cuadro1). Las otras nueve especies fueron escasas, menos del $1 \%$ del total.

Con respecto a la altitud, L. youngifue la especie predominante en los pisos altitudinales investigados de los 600 a los $1.400 \mathrm{msnm}$, abundando, principalmente, a una altitud de 1.360 msnm; no se evidenció su presencia en la localidad de Monte Carmelo II a 1.600 msnm, a pesar de haberse realizado doce capturas a lo largo de un año de estudio. En lo que a L. ovallesi se refiere su distribución coincide con $L$. youngia excepción de la localidad ubicada a $1.400 \mathrm{msnm}$,

Cuadro 1. Distribución de especies, diversidad y abundancia según la altitud de cada localidad estudiada del municipio Andrés Eloy Blanco.

\begin{tabular}{|c|c|c|c|c|c|c|c|c|c|c|c|c|c|}
\hline Población/altitud & $\begin{array}{c}\text { L. } \\
\text { youngi }\end{array}$ & ovallesi & $\begin{array}{l}\text { L. } \\
\text { lichyi }\end{array}$ & $\begin{array}{c}\text { L. } \\
\text { scorzai }\end{array}$ & $\begin{array}{l}\text { L. } \\
\text { gomezi }\end{array}$ & $\begin{array}{c}L . \\
\text { i venezuelensis }\end{array}$ & $\begin{array}{l}\text { L. } \\
\text { sp. } c a\end{array}$ & $\begin{array}{l}L . \\
\text { ayenensis }\end{array}$ & L trinidadensis & L dubitans & $\begin{array}{c}\text { L. } \\
\text { walkeri }\end{array}$ & $\begin{array}{c}L . \\
B . s p\end{array}$ & $\begin{array}{r}L . \\
\text { Total }\end{array}$ \\
\hline Represa/600 m & 0 & 0 & 0 & 0 & 0 & 0 & 0 & 0 & 0 & 0 & 0 & 0 & 0 \\
\hline Yacambu/ $600 \mathrm{~m}$ & 9 & 25 & 0 & 0 & 0 & 0 & 0 & 4 & 0 & 0 & 0 & 11 & 49 \\
\hline Yacambu/ 800 m & 15 & 12 & 0 & 0 & 8 & 0 & 0 & 0 & 0 & 0 & 0 & 11 & 46 \\
\hline Yacambu/1.000 m & 46 & 51 & 0 & 14 & 0 & 2 & 0 & 0 & 0 & 0 & 0 & 66 & 179 \\
\hline Las Virtudes/1.000 m & 2 & 0 & 0 & 0 & 1 & 0 & 0 & 0 & 0 & 0 & 0 & 0 & 3 \\
\hline Sanare/1.180 m & 43 & 3 & 5 & 0 & 1 & 0 & 0 & 0 & 2 & 0 & 0 & 0 & 54 \\
\hline Periquito/1.200 m & 3 & 0 & 1 & 0 & 0 & 1 & & 0 & 0 & 0 & 0 & 0 & 5 \\
\hline La Escalera/1.250 m & 9 & 4 & 0 & 2 & 0 & 0 & 0 & 0 & 0 & 0 & 0 & 0 & 15 \\
\hline Las Maticas/1.360 m & 9486 & 285 & 34 & 0 & 0 & 0 & 5 & 0 & 0 & 2 & 1 & 18 & 9.831 \\
\hline Monte Carmelo/1.400 m & n 136 & 0 & 5 & 0 & 0 & 0 & 0 & 0 & 0 & 0 & 0 & 3 & 144 \\
\hline Monte Carmelo/1.600 m & n 0 & 0 & 0 & 0 & 0 & 0 & 0 & 0 & 0 & 0 & 0 & 0 & 0 \\
\hline Total & 9749 & 380 & 45 & 16 & 10 & 3 & 5 & 4 & 2 & 2 & 1 & 1091 & 10.326 \\
\hline
\end{tabular}


es decir, un rango de dispersión desde los 600 msnm hasta los 1.360 msnm., con mayor abundancia que $L$. youngipor debajo de los 1.000 msnm.

En lo que respecta al registro de las otras nueve especies $(<1 \%)$, se encontró que su presencia estuvo asociada a ciertas altitudes es así como de los 45 ejemplares capturados de L. lichyi (Floch y Abonnenc, 1950) 34 de ellos fueron recolectados a 1.360 msnm, en Las Maticas; L. scorzai (Ortiz, 1965) de 16 ejemplares, 14 de ellos a $1.000 \mathrm{msnm}$ y L. gomezi (Nitzulescu, 1931) de 10 ejemplares capturados ocho fueron atrapados a $800 \mathrm{msnm}$. En la población de Las Maticas se recolectaron 9.831 ejemplares, con una diversidad de siete especies a saber L. youngi, L. ovallesi, L. scorzai, L. walkeri (Newstead, 1914), L. dubitans (Sherlock, 1962), Lutzomyia sp. y Brumptomyia sp.

Con respecto a la variación de la población, se determinó que la especie predominante fue $L$. youngique presentó un pico de mayor abundancia al final de la estación seca de un régimen unimodal, recolectándose 2.786 ejemplares solamente en esta captura/mes. En la figura $1 \mathrm{se}$ observa que el pico de abundancia coincidió con la mayor temperatura media mensual, $24^{\circ} \mathrm{C}$, y una humedad relativa mensual de $70 \%$ la cual fue la más baja registrada en el año.

Esta variación de la población de L. youngi en Las Maticas, además, fue seguida en función de la actividad durante las primeras tres horas de la noche (figura 2), evidenciándose que su mayor actividad fue entre las 19:30 y las 20:30 horas, disminuyendo progresivamente en $50 \%$ de las 20:40 a las 22:50 horas, principalmente, entre los meses de diciembre a mayo. La actividad de $L$. youngi en Las Maticas fue mayor en el ambiente peridoméstico (principalmente, en febrero, marzo y abril) seguido del área selvática (con mayor abundancia entre mayo y septiembre, noviembre y diciembre) y, por último, en el ambiente intradomiciliario (figura 3).

Con relación a las 8.867 hembras disecadas, ninguna de ellas presentó flagelados.

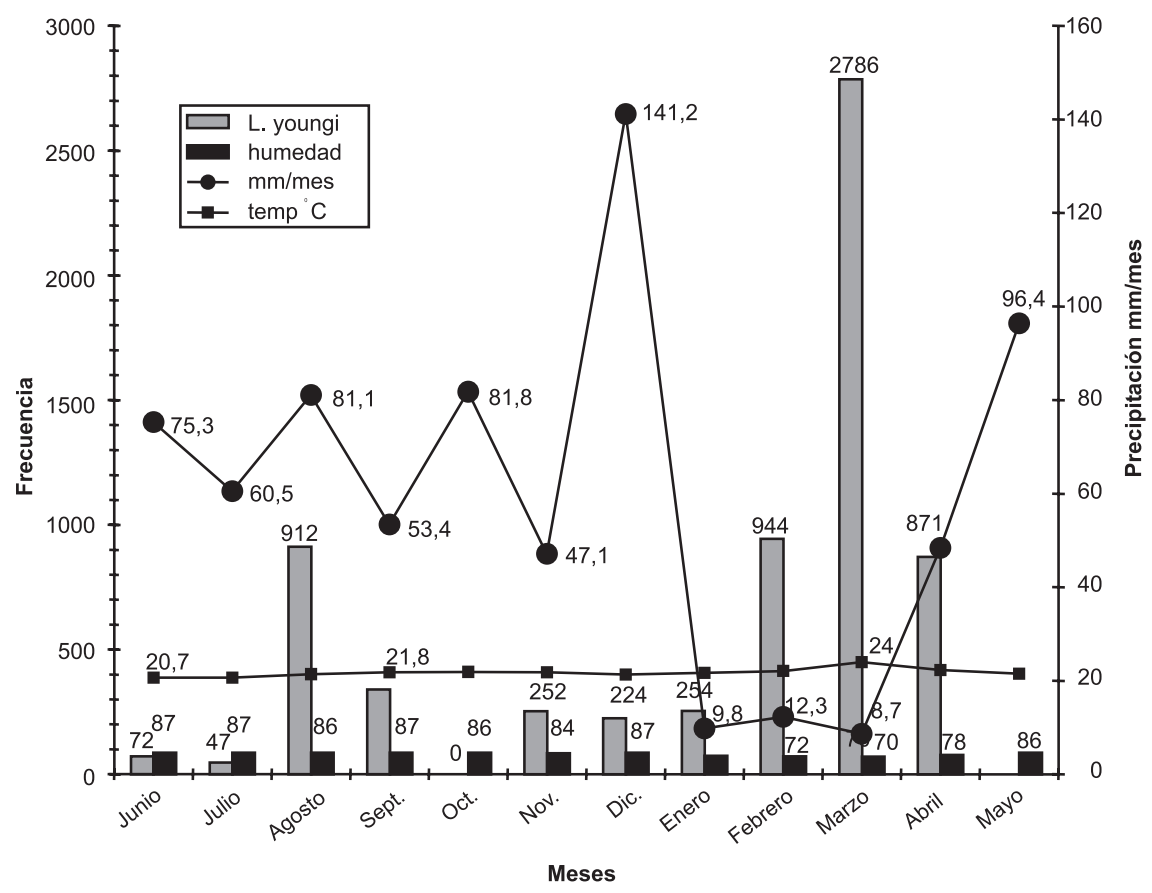

Figura 1. Frecuencia mensual de Lutzomyia youngi en la población de Las Maticas (trampas Shannon y CDC) y su relación con la precipitación, humedad relativa y temperatura. 


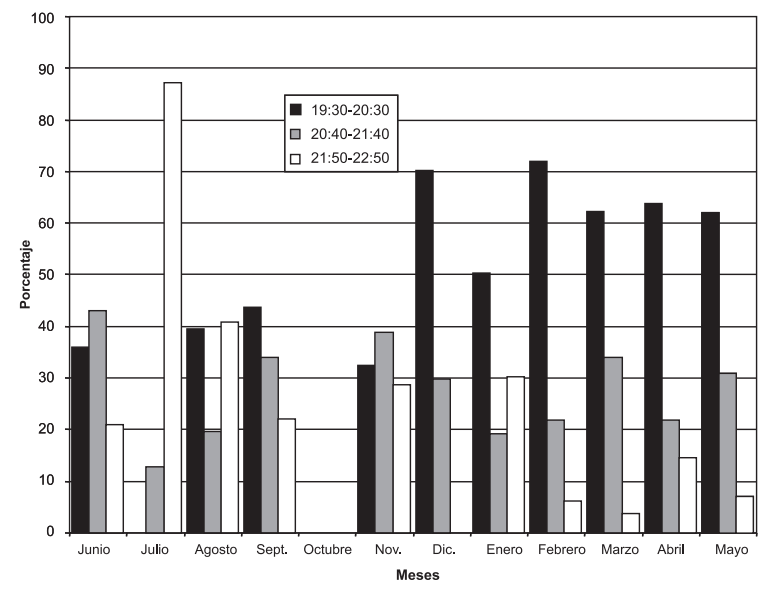

Figura 2. Actividad horaria mensual de Lutzomyia youngi en la población de Las Maticas expresada en porcentajes, a las 19:30-20:30 horas (negro), 20:40-21:40 horas (gris) y 21:50-22:50 horas (blanco).

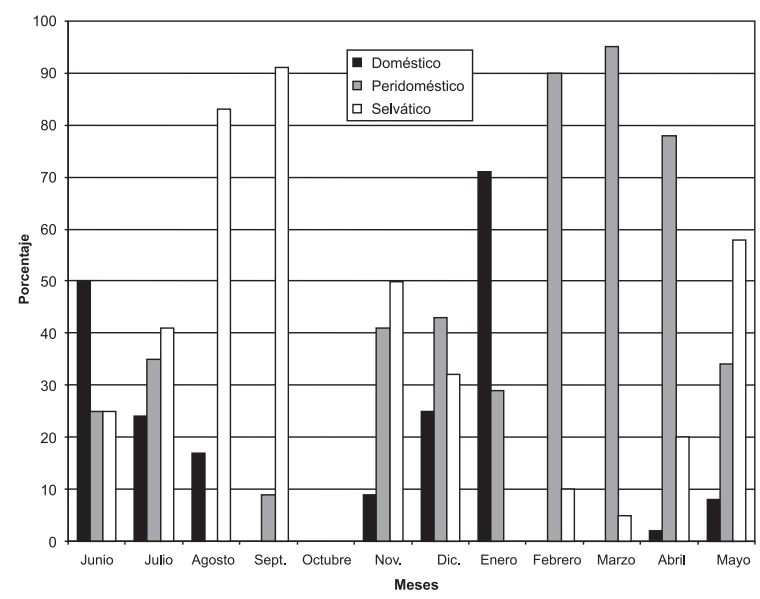

Figura 3. Porcentajes mensuales de abundancia de Lutzomyia youngi en la población de Las Maticas en ambientes doméstico (negro), peridoméstico (gris) y selvático (blanco).

\section{Discusión}

Con la trampa Shannon se lograron capturas más numerosas que con las CDC; no se hicieron capturas sobre cebo humano para disminuir el riesgo de infección. En ambas trampas la especie que más abundó en todos los puntos de captura fue L. youngi; también, fue la especie con mayor dispersión ya que se consiguió en todos los pisos altitudinales estudiados (desde los 600 hasta los $1.400 \mathrm{msnm}$ ) tal como lo refieren otros autores
(5-7). De aquí que, en el estado Trujillo, señalen a L. youngi como especie que predomina sobre los $800 \mathrm{msnm}$ hasta los $1.700 \mathrm{msnm}$, mientras que L. ovallesi lo refieren entre los 200 y los 1.800 msnm (8).

En el estado Mérida reportan a L. youngi como la especie más abundante en todos los pisos altitudinales estudiados (superior al 58\%), seguido de L. trinidadensis (Newst, 1922) y de L. ovallesi $(9,10)$. Al nordeste del estado Táchira en los límites con el estado Mérida describen a $L$. youngi como la especie más abundante y extensa del occidente venezolano (11). En América esta especie se ha reportado en Costa Rica y en Colombia, donde se señala en el Valle del Cauca como la más abundante con $73 \%$ de los ejemplares capturados, seguido de $L$. lichyi con $21 \%(12,13)$.

Esto sugiere la importancia de esta especie en la zona, seguido de $L$. ovallesi, aunque menor en abundancia (3,72\%); su característica de ser altamente antropofílica (6) y de haber sido encontrada naturalmente infectada $(3,14)$, la convierten en el segundo probable vector de leishmaniasis en la zona, específicamente, en pisos iguales o inferiores a los $1.000 \mathrm{msnm}$., donde $L$. ovallesi fue más numerosa en las capturas que L. youngi, situación presentada también en el estado Anzoátegui en áreas de leishmaniasis cutánea y con una diversidad de 18 especies; aquí reportan a $L$. ovallesi también con total predominio por debajo de los $1.000 \mathrm{msnm}$, específicamente, entre los 790 y $886 \mathrm{msnm}$, sin que se presente $L$. youngi a pesar de la diversidad $(15,16)$.

Por otro lado, en el estado Bolívar (Venezuela) con una diversidad de 18 especies, L. ovallesies reportada con apenas $0,2 \%$ de las capturas, mientras que $L$. youngi tampoco se identificó (17), lo cual reafirma el carácter limitado de $L$. youngi en los Andes venezolanos (Táchira, Mérida, Trujillo y sur de Lara) entre los 760 a 1.800 msnm (3).

Otras especies capturadas fueron: $L$. gomezi y $L$. lichyi de hábitos antropofílicos y L. cayennensis (Floch y Abonnenc, 1941), L. venezuelensis (Floch y Abonnenc, 1948), L. scorzai, L. trinidadensis y Brumptomyia sp. Otro elemento interesante fue el hallazgo de $L$. walkeri, especie que no había sido descrita para el estado Lara. 
Con respecto a la actividad de $L$. youngi, se tiene que desde las 18:30 hasta las 19:30 es -de las tres horas estudiadas en trampa Shannon- cuando se presenta mayor riesgo de picadura por este flebótomo. Esta mayor actividad en las primeras horas de la noche ha sido descrita anteriormente en una zona próxima a Cali a $1.375 \mathrm{msnm}$, señalando que $L$. youngipresentaba un aumento proporcional desde las 19:00 horas hasta las 21:00 horas (máxima abundancia) (13). En el estado Miranda, utilizando trampa Shannon, se señaló un incremento de $L$. ovallesi entre las $18: 00$ y las 20:00 horas y en Trujillo se describió a $L$. ovallesi con un pico de abundancia de las 24:00 a las 01:00 $(7,18)$. Todo esto para indicar que existen horas de mayor actividad para cada especie, particularmente coincidiendo esta actividad con las horas crepusculares.

Con respecto al estudio de las zonas domésticas (10\% del total de ejemplares), peridomésticas (62\%) y selváticas (28\%) con trampas CDC, se tiene que las zonas peridomésticas (gallineros) fueron las que presentaron mayor abundancia de L. youngi en todas las poblaciones estudiadas, con 535 ejemplares, seguido de 238 y 86 especímenes en las áreas selváticas y domésticas, respectivamente. Esta abundancia de $L$. youngien los ambientes peridomésticos en Andrés Eloy Blanco es influenciada por la atracción que ejercen las aves de corral y otros animales peridomésticos sobre los flebótomos, elemento descrito en Brasil donde se observa que las aves y sus plumas son un poderoso atrayente para los flebótomos, especialmente, L. migonei (França, 1920) y L. pessoai (Coutinho y Barreto, 1940) (19).

Desde el punto de vista climático, otro elemento que se apreció fue el máximo pico de abundancia que ocurría en febrero, marzo y abril, especialmente, en marzo, cuando tanto en las trampas Shannon como CDC, hubo un máximo de capturas para $L$. youngi. Este pico máximo coincidió, en primer lugar, con el comienzo de las lluvias después del período de verano; en segundo lugar con el período de mayor temperatura media mensual $\left(24^{\circ} \mathrm{C}\right)$; en tercer lugar, con el mes de menor humedad (70\%) y, en cuarto lugar, con el mes cuando hubo menos precipitaciones. Por el contrario, cuando las precipitaciones sobre- pasaron los $47 \mathrm{~mm} / \mathrm{mes}$, las poblaciones de $L$. youngi se mantuvieron muy bajas (excepto en agosto cuando hubo un pequeño pico).

Esta relación entre elementos climáticos y abundancia de flebótomos ha sido descrita también para L. youngi en Colombia señalando primero un pico de abundancia en agosto que coincide con el final del período seco y el comienzo de las primeras lluvias para, luego, caer la abundancia cuando estas lluvias sobrepasaban los $90 \mathrm{~mm}$ y, en segundo lugar, que los picos nocturnos coincidían con la disminución de la temperatura y el aumento de la humedad (13); posteriormente, en la población de Las Calderas (1.350 msnm) del estado Trujillo, en una zona próxima a áreas endémicas de leishmaniasis cutánea americana, se señaló la existencia de altas poblaciones de L. youngi las cuales presentaron dos picos de abundancia parecidos a los de Andrés Eloy Blanco, uno en abril $(90 \mathrm{~mm} /$ mes) y otro en agosto (31 mm/mes), apreciándose un mínimo en diciembre cuando las lluvias sobrepasaron los $120 \mathrm{~mm} / \mathrm{mes}$ (20), simultáneamente relacionaron la abundancia con el comienzo del período de las lluvias.

En el estado Táchira se señala que los casos de leishmaniasis cutánea americana aumentan considerablemente en los dos meses que siguen a las primeras lluvias después del verano, tal vez porque el pico de flebótomos también coincidió con las primeras lluvias luego del verano, presentándose los casos un mes después del contacto hombre-vector infectado (11).

Es importante lo discutido por otros autores que proponen que el aumento súbito de la abundancia de flebótomos corresponde a un aumento de la eclosión de las pupas; igualmente, la disminución que ocurre cuando la precipitación es máxima es el resultado catastrófico de las lluvias sobre las larvas de los flebótomos (21). Basado en esto, pareciera que en la población de Las Maticas, las larvas después del intenso período seco con altas temperaturas y baja humedad, esperan las primeras lluvias del invierno para despertar el reloj biológico de la eclosión de las pupas.

Con respecto a la abundancia y distribución de especies en los distintos pisos de captura, se tiene 
que la mayor abundancia se localizó en la población de Las Maticas: $1.320 \mathrm{msnm}, 18,7^{\circ} \mathrm{C}$, pluviosidad media anual de $1.200 \mathrm{~mm}$ y bosque seco premontano; esta población coincide con otra abundancia de $L$. youngia $1.375 \mathrm{msnm}$, reportada en la población de La Guaira, departamento del Valle del Cauca, Colombia (13) y a la descripción en la población de Las Calderas, Trujillo, prácticamente a la misma altura $(1.350 \mathrm{msnm})$, la cual se caracteriza por presentar también abundancia de $L$. youngi, lo cual sugiere que los meses más secos como los mejores para hacer las capturas (21). Esto coincide con los hallazgos de este trabajo, en el que se encuentra un pico en el mes más caliente y seco que coincide con las primeras lluvias.

La segunda población que tuvo mayor abundancia de L. youngifue Monte Carmelo, a 1.400 msnm., seguido de Sanare 1.180 msnm. y Yacambú 1.000 msnm, pero ya en esta última altura $L$. ovallesi presentaba mayor abundancia que $L$. youngi, lo que podría sugerir que esta zona, entre los 1.180 m. y los $1.400 \mathrm{msnm}$, es la altitud donde hay mayor abundancia de L. youngi, con una limitante a partir de los $1.600 \mathrm{msnm}$, altura a partir de la cual no se detectó presencia de ninguna especie de flebótomos y por debajo de los $1.000 \mathrm{msnm}$, donde L. ovallesi podría ser por predominio, el vector incriminado en la transmisión.

Otro elemento que resaltó fue las capturas en la población de Monte Carmelo que es la segunda en aporte de casos en el municipio y se caracteriza por presentar un relieve con pronunciadas pendientes; aquí se hicieron capturas a $1.400 \mathrm{msnm}$ y a $1.600 \mathrm{msnm}$; la distancia que separaba a ambos puntos por la carretera era de aproximadamente $2.000 \mathrm{~m}$. Sin embargo, a los $1.400 \mathrm{msnm}$, las capturas fueron exitosas, mientras que a $1.600 \mathrm{msnm}$, y a lo largo de un año, no se capturó ningún flebótomo. Es de resaltar que en la zona alta (1.600 msnm) nunca se habían presentado casos, lo cual indica que a los $1.600 \mathrm{msnm}$ de altitud se presenta una limitante para todos los flebótomos, especialmente, para L. youngi y, por ende, una barrera para la enfermedad. Esta relación también la indican en Trujillo, refiriendo que a partir de 1.600 y hasta los 1.900 msnm disminuía la población de
L. youngi (8) y, posteriormente, en el estado Mérida se señala un límite de altura de 1.600 msnm para la captura de $L$. youngi $(9,10)$.

Otra característica importante es que en la mayoría de los poblados de este municipio donde se reportaban casos de leishmaniasis cutánea, señalaban temperaturas medias anuales iguales o inferiores a $19^{\circ} \mathrm{C}$; estas temperatura son descritas por algunos autores como una limitante en la transmisión de la enfermedad (22). Sin embargo en febrero, marzo y abril las temperaturas aumentan hasta $4^{\circ} \mathrm{C}$ por encima de la media anual, temperaturas en las cuales no hay limitantes por la isoterma de $19^{\circ} \mathrm{C}$ siendo en los meses más calurosos cuando se determinó mayor abundancia de L. youngi. Por lo tanto, en esta época se presenta el momento ideal para la transmisión de la enfermedad y, por consiguiente, el siguiente trimestre (mayo, junio, julio) se identifica por los pobladores como los meses cuando hay mayor número de casos, habiendo transcurrido el tiempo de incubación de la enfermedad y coincidiendo esto con el período de lluvias (figura 1), incrementándose la asociación lluvia-leishmaniasis que establecen los habitantes del municipio.

La ausencia de hembras infectadas con promastigotes impidió la identificación de las posibles especies de Leishmania implicadas en la transmisión; sin embargo, en el estado Trujillo ya se habían señalado 4 de 1.872 hembras de $L$. townsendi (Ortiz, 1959) infectadas con L. braziliensis (Vianna, 1911). Es necesario señalar que $L$. youngi era descrita en estas zonas como L. townsendiantes de $1987(3,23)$.

En conclusión, se tiene que $L$. youngi es la especie más abundante de las 11 descritas, con un predominio a los $1.320 \mathrm{msnm}$ y en febrero, marzo y abril, específicamente, cuando la temperatura media mensual es máxima y la humedad y las precipitaciones mensuales son mínimas, lo cual permite potenciar las medidas preventivas (uso de insecticidas, mosquiteros, etc.) en las zonas y en los meses descritos, logrando un uso más racional de los recursos e influyendo directamente en el control y la disminución de la transmisión de la leishmaniasis cutánea americana. 


\section{Agradecimientos}

Al personal de la Sección de Parasitología Médica de la UCLA, al personal del Laboratorio de Biología de Lutzomyia (Centro JWT) ULA, Trujillo; al personal del Ministerio del Ambiente-Lara y a las familias Escalona, Pérez, Mendoza, Jiménez y Betancourt en el municipio Andrés Eloy Blanco por su valiosa colaboración.

\section{Conflicto de intereses}

Se tuvo acceso irrestricto a todos los datos del presente estudio y asumo plena responsabilidad por la integridad de los datos y la exactitud del análisis.

\section{Fuentes de financiación}

El financiamiento provino del Concejo de Desarrollo Científico y Humanístico de la Universidad Centroccidental "Lisandro Alvarado", CDCHT-UCLA (suministro de equipos y reactivos) y de la Dirección de Formación de Personal Académico del Vicerrectorado Académico de la UCLA.

\section{Referencias}

1. Arredondo CC. Phlebotominae de Venezuela: Lutzomyia amilcari nsp. del Estado Lara. Mem Inst Oswaldo Cruz 1984;79:63-9.

2. Bonfante-Garrido R, Urdaneta I, Alvarado J, Anzola NH, Torrealba J, Saldivia ME et al. Phlebotomine sand flies in five endemic leishmaniasis foci in Lara State, Venezuela. Bol Dir Malariol San Amb 1995;35(Supl.1):53-62.

3. Feliciangeli MD, Rodríguez N, De Guglielmo Z, Rodríguez A. La fauna flebotómica (Diptera, Psychodidae) en Venezuela. I.Taxonomía y distribución geográfica. Bol Dir Malariol San Amb 1988;28:99-113.

4. Young DG, Duncan MA. Guide to the identification and geographic distribution of Lutzomyia sand flies in Mexico the West Indies, Central and South America (Diptera: Psychodidae). Mem Am Entomol Inst 1994;54:1-881.

5. Ministerio del Ambiente y de los Recursos Naturales. Anuario hidrometeorológico estado Lara. Años 2001-2002. El Carabalí: Ministerio del Ambiente y de los Recursos Naturales; 2003.

6. Feliciangeli MD, Arredondo C, Ward R. Phlebotomine sand flies in Venezuela review of the verrucarum species group (in part) of Lutzomyia (Diptera: Psychodidae) with description of a new species from Lara. J Med Entomol 1992;29:729-44.
7. Feliciangeli MD, Reyes RM, Limongi JE. Natural infection of Lutzomyia ovallesi (Diptera: Psychodidae) with parasites of the Leishmania braziliensis complex in an restricted focus of cutaneous leishmaniasis in northern Venezuela. Mem Inst Oswaldo Cruz 1988;83:393-4.

8. Mogollón J, Manzanilla P, Scorza JV. Distribución altitudinal de nueve especies de Lutzomyia (Diptera, Psychodidae) en el estado Trujillo, Venezuela. Bol Dir Malariol San Amb 1977;17:206-23.

9. Añez N, Cazorla D, Nieves E, Chataing B, Castro M, de Yarbuh AL. Epidemiología de la leishmaniasis cutánea en el estado Mérida. Venezuela. Diversidad y dispersión de especies flebotominas en tres pisos altitudinales y su posible rol en la transmisión de la enfermedad. Mem Inst Oswaldo Cruz 1988;83:455-63.

10. Añez N, Nieves E, Cazorla D, Oviedo M, de Yarbuh AL, Valera M. Epidemiology of cutaneous leishmaniasis in Mérida, Venezuela. III. Altitudinal distribution, age structure, natural infection and feeding behaviour of sand flies and their relation to the risk of transmission. Ann Trop Med Parasitol 1994;88:279-87.

11. Maingon R, Feliciangeli MD, Guzmán B, Rodríguez $\mathrm{N}$, Convit J, Adamson R et al. Cutaneous leishmaniasis in Táchira State, Venezuela. Ann Trop Med Parasit 1994;88:29-36.

12. Murillo J, Zeledon R. Flebótomos de Costa Rica. Brenesia 1985;23:1-137.

13. Alexander B, Usma MC, Cadena H, Quesada BL, Solarte Y, Roa W et al. Phlebotomine sandflies associated with a focus of cutaneous leishmaniasis in Valle del Cauca, Colombia. Med Vet Entomol 1995;9:273-8.

14. Bonfante-Garrido R, Urdaneta R, Urdaneta I, Alvarado J. Natural infection of Lutzomyia ovallesi (Diptera: Psychodidae) with Leishmania in Duaca, Lara State, Venezuela. Trans R Soc Trop Med Hyg 1991;85:61.

15. González R, Ledezma E, Jonquera A, De Souza L, Devera R. Flebotomofauna en áreas endémicas de leishmaniasis a distintos niveles altitudinales. Estado Anzoátegui, Venezuela. Bol Dir Malariol San Amb 1995;35:147-54.

16. González R, Jonquera A, De Sousa L, Ledesma E, Devera R. Sandfly fauna of endemic leishmaniasis foci in Anzoátegui State, Venezuela. Trans R Soc Trop Med Hyg 2002;96:57-9.

17. González R, Devera R. Fauna flebotomínica (Diptera, Psychodidae, Phlebotominae) do Estado Bolívar, Venezuela. Rev Soc Bras Med Trop 1999;32:721-3.

18. Traviezo L, Hernández D, Barreto K, Vívenes A, González A, Barazarte $\mathbf{R}$ et al. Comportamiento alternativo de dos especie de flebótomos en la comunidad de Montañas de Peraza, Municipio Pampan. Estado Trujillo. Venezuela. Acta Científica Venezolana 2001;1:125. 
19. Blazius R. A chicken pen as a breeding place for sand flies. Entomología y Vectores 2002;9:102.

20. Álvarez L, Scorza JV. Determinación de épocas de capturas de Lutzomyia youngi en Calderas, Trujillo, Venezuela. Revista Talleres 1999;6:122-3.

21. Scorza JV, Rojas E. Actividad intradomiciliar de Lutzomyia youngi (Diptera, Psychodidae) en Venezuela. Bol Dir Malariol San Amb 1989;29:64-70.
22. Scorza JV, Márquez JC, Márquez M. La isoterma de $19^{\circ} \mathrm{C}$ como factor limitante de la endemicidad de la leishmaniasis cutánea en los Andes de Venezuela. Bol Dir Malariol San Amb 1986;26:42-9.

23. Scorza J, Márquez M, Márquez JC. Hallazgo de Lutzomyia townsendi (Ortiz, 1959) naturalmente infectada con Leishmania braziliensis, en el área suburbana de Trujillo, Venezuela. Bol Dir Malariol San Amb 1984;24:21-8. 